\title{
A new iterative scheme with nonexpansive mappings for equilibrium problems
}

Anh $\mathrm{PN}^{1^{*}}$ and Thanh $\mathrm{DD}^{2}$

\footnotetext{
* Correspondence: anhpn@ptit.edu. vn

${ }^{1}$ Department of Scientific Fundamentals, Posts and Telecommunications Institute of Technology, Hanoi, Vietnam Full list of author information is available at the end of the article
}

\begin{abstract}
In this paper, we suggest a new iteration scheme for finding a common of the solution set of monotone, Lipschitz-type continuous equilibrium problems and the set of fixed points of a nonexpansive mapping. The scheme is based on both hybrid method and extragradient-type method. We obtain a strong convergence theorem for the sequences generated by these processes in a real Hilbert space. Based on this result, we also get some new and interesting results. The results in this paper generalize, extend, and improve some well-known results in the literature.

AMS 2010 Mathematics subject classification: 65 K10, 65 K15, 90 C25, 90 C33.

Keywords: Equilibrium problems, nonexpansive mappings, monotone, Lipschitz-type continuous, fixed point
\end{abstract}

\section{Introduction}

Let $\mathcal{H}$ be a real Hilbert space with inner product $\langle\cdot, \cdot\rangle$ and norm $\|\cdot\|$. Let $C$ be a nonempty closed convex subset of a real Hilbert space $\mathcal{H}$. A mapping $S: C \rightarrow C$ is a contraction with a constant $\delta \in(0,1)$, if

$$
\|S(x)-S(y)\| \leq \delta\|x-y\|, \forall x, y \in C .
$$

If $\delta=1$, then $S$ is called nonexpansive on $C$. Fix $(S)$ is denoted by the set of fixed points of $S$. Let $f: C \times C \rightarrow \mathcal{R}$ be a bifunction such that $f(x, x)=0$ for all $x \in C$. We consider the equilibrium problem in the sense of Blum and Oettli (see [1]) which is presented as follows:

$$
\text { Find } x^{*} \in C \text { such that } f\left(x^{*}, y\right) \geq 0 \text { for all } y \in C . \quad E P(f, C)
$$

The set of solutions of $E P(f, C)$ is denoted by $\operatorname{Sol}(f, C)$. The bifunction $f$ is called strongly monotone on $C$ with $\beta>0$, if

$$
f(x, y)+f(y, x) \leq-\beta\|x-y\|^{2}, \forall x, y \in C ;
$$

monotone on $C$, if

$$
f(x, y)+f(y, x) \leq 0, \forall x, y \in C ;
$$

pseudomonotone on $C$, if

$$
f(x, y) \geq 0 \text { implies } f(y, x) \leq 0, \forall x, y \in C \text {; }
$$


Lipschitz-type continuous on $C$ with constants $c_{1}>0$ and $c_{2}>0$ (see [2]), if

$$
f(x, y)+f(y, z) \geq f(x, z)-c_{1}\|x-y\|^{2}-c_{2}\|y-z\|^{2}, \forall x, y, z \in C .
$$

It is well-known that Problem $E P(f, C)$ includes, as particular cases, the optimization problem, the variational inequality problem, the Nash equilibrium problem in noncooperative games, the fixed point problem, the nonlinear complementarity problem and the vector minimization problem (see [2-6]).

In recent years, the problem to find a common point of the solution set of problem $(E P)$ and the set of fixed points of a nonexpansive mapping becomes an attractive field for many researchers (see [7-15]). An important special case of equilibrium problems is the variational inequalities (shortly $(V I P)$ ), where $F: C \rightarrow \mathcal{H}$ and $f(x, y)=\langle F(x), y$ $x\rangle$. Various methods have been developed for finding a common point of the solution set of problem (VIP) and the set of fixed points of a nonexpansive mapping when $F$ is monotone (see [16-18]).

Motivated by fixed point techniques of Takahashi and Takahashi in [19] and an improvement set of extragradient-type iteration methods in [20], we introduce a new iteration algorithm for finding a common of the solution set of equilibrium problems with a monotone and Lipschitz-type continuous bifunction and the set of fixed points of a nonexpansive mapping. We show that all of the iterative sequences generated by this algorithm convergence strongly to the common element in a real Hilbert space.

\section{Preliminaries}

Let $C$ be a nonempty closed convex subset of a Hilbert space $\mathcal{H}$. We write $x^{n} \rightarrow x$ to indicate that the sequence $\left\{x^{n}\right\}$ converges weakly to $x$ as $n \rightarrow \infty, x^{n} \rightarrow x$ implies that $\left\{x^{n}\right\}$ converges strongly to $x$. For any $x \in \mathcal{H}$, there exists a nearest point in $C$, denoted by $\operatorname{Pr}_{C}(x)$, such that

$$
\left\|x-\operatorname{Pr}_{C}(x)\right\| \leq\|x-y\|, \forall y \in C .
$$

$\operatorname{Pr}_{C}$ is called the metric projection of $\mathcal{H}$ to $C$. It is well known that $\operatorname{Pr}_{C}$ satisfies the following properties:

$$
\begin{aligned}
& \left\langle x-y, \operatorname{Pr}_{C}(x)-\operatorname{Pr}_{C}(y)\right\rangle \geq\left\|\operatorname{Pr}_{C}(x)-\operatorname{Pr}_{C}(y)\right\|^{2}, \forall x, y \in \mathcal{H}, \\
& \left\langle x-\operatorname{Pr}_{C}(x), \operatorname{Pr}_{C}(x)-y>\right\rangle \geq 0, \forall x \in \mathcal{H}, y \in C, \\
& \|x-y\|^{2} \geq\left\|x-\operatorname{Pr}_{C}(x)\right\|^{2}+\left\|y-\operatorname{Pr}_{C}(x)\right\|^{2}, \forall x \in \mathcal{H}, y \in C .
\end{aligned}
$$

Let us assume that a bifunction $f: C \times C \rightarrow \mathcal{R}$ and a nonexpansive mapping $S: C \rightarrow$ $C$ satisfy the following conditions:

$A_{1} . f$ is Lipschitz-type continuous on $C$;

$A_{2} . f$ is monotone on $C$;

$A_{3}$. for each $x \in C, f(x, \cdot)$ is subdifferentiable and convex on $C$;

$A_{4} . \operatorname{Fix}(S) \cap \operatorname{Sol}(f, C) \neq \varnothing$.

Recently, Takahashi and Takahashi in [19] first introduced an iterative scheme by the viscosity approximation method. The sequence $\left\{x^{k}\right\}$ is defined by: 


$$
\left\{\begin{array}{l}
x^{0} \in \mathcal{H} \\
\text { Find } u^{k} \in C \text { such that } f\left(u^{k}, y\right)+\frac{1}{r_{k}}\left\langle y-u^{k}, u^{k}-x^{k}\right\rangle \geq 0, \quad \forall y \in C, \\
x^{k+1}=\alpha_{k} g\left(x^{k}\right)+\left(1-\alpha_{k}\right) S\left(u^{k}\right), \forall k \geq 0,
\end{array}\right.
$$

where $C$ is a nonempty closed convex subset of $\mathcal{H}$ and $g$ is a contractive mapping of $\mathcal{H}$ into itself. The authors showed that under certain conditions over $\left\{\alpha_{k}\right\}$ and $\left\{r_{k}\right\}$, sequences $\left\{x_{k}\right\}$ and $\left\{u_{k}\right\}$ converge strongly to $z=\operatorname{Pr}_{\operatorname{Sol}(f, C) \cap F i x(S)}\left(g\left(x^{0}\right)\right)$. Recently, iterative methods for finding a common element of the set of solutions of equilibrium problems and the set of fixed points of a nonexpansive mapping have further developed by many authors. These methods require to solve approximation auxilary equilibrium problems.

In this paper, we introduce a new iteration method for finding a common point of the set of fixed points of a nonexpansive mapping $S$ and the set of solutions of problem $E P(f, C)$. At each our iteration, the main steps are to solve two strongly convex problems

$$
\left\{\begin{array}{l}
y^{k}=\operatorname{argmin}\left\{\lambda_{k} f\left(x^{k}, y\right)+\frac{1}{2}\left\|y-x^{k}\right\|^{2}: y \in C\right\} \\
t^{k}=\operatorname{argmin}\left\{\lambda_{k} f\left(y^{k}, y\right)+\frac{1}{2}\left\|y-x^{k}\right\|^{2}: y \in C\right\}
\end{array}\right.
$$

and compute the next iteration point by Mann-type fixed points

$$
x^{k+1}=\alpha_{k} g\left(x^{k}\right)+\left(1-\alpha_{k}\right) S\left(t^{k}\right),
$$

where $g: C \rightarrow C$ is a $\delta$-contraction with $0<\delta<\frac{1}{2}$.

To investigate the convergence of this scheme, we recall the following technical lemmas which will be used in the sequel.

Lemma 2.1 (see [21]) Let $\left\{a_{n}\right\}$ be a sequence of nonnegative real numbers such that:

$$
a_{n+1} \leq\left(1-\alpha_{n}\right) a_{n}+\beta_{n}, n \geq 0,
$$

where $\left\{\alpha_{n}\right\}$, and $\left\{\beta_{n}\right\}$ satisfy the conditions:

(i) $\alpha_{n} \subset(0,1)$ and $\sum_{n=1}^{\infty} \alpha_{n}=\infty$;

(ii) $\limsup _{n \rightarrow \infty} \frac{\beta_{n}}{\alpha_{n}} \leq 0$ or $\sum_{n=1}^{\infty}\left|\beta_{n}\right|<\infty$.

Then

$$
\lim _{n \rightarrow \infty} a_{n}=0 .
$$

Lemma 2.2 ([22]) Assume that $S$ is a nonexpansive self-mapping of a nonempty closed convex subset $C$ of a real Hilbert space $\mathcal{H}$. If Fix $(S) \neq \varnothing$, then $I-S$ is demiclosed; that is, whenever $\left\{x^{k}\right\}$ is a sequence in $C$ weakly converging to some $\bar{x} \in C$ and the sequence $\left\{(I-S)\left(x^{k}\right)\right\}$ strongly converges to some $\bar{\gamma}$, it follows that $(I-S)(\bar{x})=\bar{\gamma}$. Here I is the identity operator of $\mathcal{H}$.

Lemma 2.3 (see [20], Lemma 3.1) Let $C$ be a nonempty closed convex subset of a real Hilbert space $\mathcal{H}$. Let $f: C \times C \rightarrow \mathcal{R}$ be a pseudomonotone, Lipschitz-type continuous bifunction with constants $c_{1}>0$ and $c_{2}>0$. For each $x \in C$, let $f(x, \cdot)$ be convex and subdifferentiable on C. Suppose that the sequences $\left\{x^{k}\right\},\left\{y^{k}\right\},\left\{t^{k}\right\}$ generated by Scheme (2.4) and $x^{*} \in \operatorname{Sol}(f, C)$. Then

$$
\left\|t^{k}-x^{*}\right\|^{2} \leq\left\|x^{k}-x^{*}\right\|^{2}-\left(1-2 \lambda_{k} c_{1}\right)\left\|x^{k}-y^{k}\right\|^{2}-\left(1-2 \lambda_{k} c_{2}\right)\left\|\gamma^{k}-t^{k}\right\|^{2}, \forall k \geq 0 .
$$




\section{Main results}

Now, we prove the main convergence theorem.

Theorem 3.1 Suppose that Assumptions $A_{1}-A_{4}$ are satisfied, $x^{0} \in C$ and two positive sequences $\left\{\lambda_{k}\right\},\left\{a_{k}\right\}$ satisfy the following restrictions:

$$
\left\{\begin{array}{l}
\sum_{k=0}^{\infty}\left|\alpha_{k+1}-\alpha_{k}\right|<\infty \\
\lim _{k \rightarrow \infty} \alpha_{k}=0 \\
\sum_{k=0}^{\infty} \alpha_{k}=\infty \\
\sum_{k=0}^{\infty} \sqrt{\left|\lambda_{k+1}-\lambda_{k}\right|}<\infty \\
\left\{\lambda_{k}\right\} \subset[a, b] \text { for some } a, b \in\left(0, \frac{1}{L}\right), \text { where } L=\max \left\{2 c_{1}, 2 c_{2}\right\} .
\end{array}\right.
$$

Then the sequences $\left\{x^{k}\right\},\left\{y^{k}\right\}$ and $\left\{t^{k}\right\}$ generated by (2.4) and (2.5) converge strongly to the same point $x^{*}$, where

$$
x^{*}=\operatorname{Pr}_{\text {Fix }(S) \cap S o l(f, C)} g\left(x^{*}\right) .
$$

The proof of this theorem is divided into several steps.

Step 1. Claim that

$$
\lim _{k \rightarrow \infty}\left\|x^{k}-t^{k}\right\|=0
$$

Proof of Step 1. For each $x^{*} \in \operatorname{Fix}(S) \cap \operatorname{Sol}(f, C)$, it follows from $x^{k+1}=a_{k} g\left(x^{k}\right)+(1-$ $\left.a_{k}\right) S\left(t^{k}\right)$, Lemma 2.3 and $\delta \in\left(0, \frac{1}{2}\right)$ that

$$
\begin{aligned}
\left\|x^{k+1}-x^{*}\right\|^{2}= & \left\|\alpha_{k}\left(g\left(x^{k}\right)-x^{*}\right)+\left(1-\alpha_{k}\right)\left(S\left(t^{k}\right)-S\left(x^{*}\right)\right)\right\|^{2} \\
\leq & \alpha_{k}\left\|g\left(x^{k}\right)-x^{*}\right\|^{2}+\left(1-\alpha_{k}\right)\left\|S\left(t^{k}\right)-S\left(x^{*}\right)\right\|^{2} \\
= & \alpha_{k}\left\|\left(g\left(x^{k}\right)-g\left(x^{*}\right)\right)+\left(g\left(x^{*}\right)-x^{*}\right)\right\|^{2}+\left(1-\alpha_{k}\right)\left\|S\left(t^{k}\right)-S\left(x^{*}\right)\right\|^{2} \\
\leq & 2 \delta^{2} \alpha_{k}\left\|x^{k}-x^{*}\right\|^{2}+2 \alpha_{k}\left\|g\left(x^{*}\right)-x^{*}\right\|^{2}+\left(1-\alpha_{k}\right)\left\|t^{k}-x^{*}\right\|^{2} \\
\leq & 2 \delta^{2} \alpha_{k}\left\|x^{k}-x^{*}\right\|^{2}+2 \alpha_{k}\left\|g\left(x^{*}\right)-x^{*}\right\|^{2}+\left(1-\alpha_{k}\right)\left\|x^{k}-x^{*}\right\|^{2} \\
& -\left(1-\alpha_{k}\right)\left(1-2 \lambda_{k} c_{1}\right)\left\|x^{k}-y^{k}\right\|^{2}-\left(1-\alpha_{k}\right)\left(1-2 \lambda_{k} c_{2}\right)\left\|y^{k}-t^{k}\right\|^{2} \\
\leq & \left\|x^{k}-x^{*}\right\|^{2}+2 \alpha_{k}\left\|g\left(x^{*}\right)-x^{*}\right\|^{2}-\left(1-\alpha_{k}\right)\left(1-2 \lambda_{k} c_{1}\right)\left\|x^{k}-y^{k}\right\|^{2} \\
& -\left(1-\alpha_{k}\right)\left(1-2 \lambda_{k} c_{2}\right)\left\|y^{k}-t^{k}\right\|^{2} .
\end{aligned}
$$

Then, we have

$$
\begin{aligned}
\left(1-\alpha_{k}\right)\left(1-2 b c_{1}\right)\left\|x^{k}-y^{k}\right\|^{2} \leq & \left(1-\alpha_{k}\right)\left(1-2 \lambda_{k} c_{1}\right)\left\|x^{k}-y^{k}\right\|^{2} \\
\leq & \left\|x^{k}-x^{*}\right\|^{2}-\left\|x^{k+1}-x^{*}\right\|^{2}+2 \alpha_{k}\left\|g\left(x^{*}\right)-x^{*}\right\|^{2} \\
& \rightarrow 0 \text { as } k \rightarrow \infty,
\end{aligned}
$$

and

$$
\lim _{k \rightarrow \infty}\left\|x^{k}-y^{k}\right\|=0 .
$$

By the similar way, also

$$
\lim _{k \rightarrow \infty}\left\|\gamma^{k}-t^{k}\right\|=0 .
$$


Combining this, (3.1) and the inequality $\left\|x^{k}-t^{k}\right\|=\left\|x^{k}-y^{k}\right\|+\left\|y^{k}-t^{k}\right\|$, we have

$$
\lim _{k \rightarrow \infty}\left\|x^{k}-t^{k}\right\|=0
$$

Step 2. Claim that

$$
\lim _{k \rightarrow \infty}\left\|x^{k+1}-x^{k}\right\|=0 .
$$

Proof of Step 2. It is easy to see that $t^{k}=\operatorname{argmin}\left\{\frac{1}{2}\left\|t-x^{k}\right\|^{2}+\lambda_{k} f\left(y^{k}, t\right): t \in C\right\}$ if and only if

$$
0 \in \partial_{2}\left(\lambda_{k} f\left(y^{k}, y\right)+\frac{1}{2}\left\|y-x^{k}\right\|^{2}\right)\left(t^{k}\right)+N_{C}\left(t^{k}\right),
$$

where $N_{C}(x)$ is the (outward) normal cone of $C$ at $x \in C$. This means that $0=\lambda_{k} w+t^{k}-x^{k}+\bar{w}$, where $w \in \partial_{2} f\left(y^{k}, t^{k}\right)$ and $\bar{w} \in N_{C}\left(t^{k}\right)$. By the definition of the normal cone $N_{C}$ we have, from this relation that

$$
\left\langle t^{k}-x^{k}, t-t^{k}\right\rangle \geq \lambda_{k}\left\langle w, t^{k}-t\right\rangle \forall t \in C .
$$

Substituting $t=t^{k+1}$ into this inequality, we get

$$
\left\langle t^{k}-x^{k}, t^{k+1}-t^{k}\right\rangle \geq \lambda_{k}\left\langle w, t^{k}-t^{k+1}\right\rangle .
$$

Since $f(x, \cdot)$ is convex on $C$ for all $x \in C$, we have

$$
f\left(y^{k}, t\right)-f\left(y^{k}, t^{k}\right) \geq\left\langle w, t-t^{k}\right\rangle \forall t \in C, w \in \partial_{2} f\left(y^{k}, t^{k}\right) .
$$

Using this and (3.3), we have

$$
\begin{aligned}
\left\langle t^{k}-x^{k}, t^{k+1}-t^{k}\right\rangle & \geq \lambda_{k}\left\langle w, t^{k}-t^{k+1}\right\rangle \\
& \geq \lambda_{k}\left(f\left(\gamma^{k}, t^{k}\right)-f\left(y^{k}, t^{k+1}\right)\right) .
\end{aligned}
$$

By the similar way, we also have

$$
\left\langle t^{k+1}-x^{k+1}, t^{k}-t^{k+1}\right\rangle \geq \lambda_{k+1}\left(f\left(y^{k+1}, t^{k+1}\right)-f\left(y^{k+1}, t^{k}\right)\right) .
$$

Using (3.4), (3.5) and $f$ is Lipschitz-type continuous and monotone, we get

$$
\begin{aligned}
\frac{1}{2} \| x^{k+1}- & x^{k}\left\|^{2}-\frac{1}{2}\right\| t^{k+1}-t^{k} \|^{2} \\
\geq & \left\langle t^{k+1}-t^{k}, t^{k}-x^{k}-t^{k+1}+x^{k+1}\right\rangle \\
\geq & \lambda_{k}\left(f\left(y^{k}, t^{k}\right)-f\left(y^{k}, t^{k+1}\right)\right) \\
& +\lambda_{k+1}\left(f\left(y^{k+1}, t^{k+1}\right)-f\left(y^{k+1}, t^{k}\right)\right) \\
\geq & \lambda_{k}\left(-f\left(t^{k}, t^{k+1}\right)-c_{1}|| y^{k}-t^{k}\left\|^{2}-c_{2}\right\| t^{k}-t^{k+1} \|^{2}\right) \\
& +\lambda_{k+1}\left(-f\left(t^{k+1}, t^{k}\right)-c_{1}\left\|y^{k+1}-t^{k+1}\right\|^{2}-c_{2}\left\|t^{k}-t^{k+1}\right\|^{2}\right) \\
\geq & \left(\lambda_{k+1}-\lambda_{k}\right) f\left(t^{k}, t^{k+1}\right) \\
\geq & -\left|\lambda_{k+1}-\lambda_{k}\right|\left|f\left(t^{k}, t^{k+1}\right)\right| .
\end{aligned}
$$

Hence

$$
\begin{aligned}
\left\|t^{k+1}-t^{k}\right\| & \leq \sqrt{|| x^{k+1}-x^{k}||^{2}+2\left|\lambda_{k+1}-\lambda_{k}\right|\left|f\left(t^{k}, t^{k+1}\right)\right|} \\
& \leq\left\|x^{k+1}-x^{k}\right\|+\sqrt{2\left|\lambda_{k+1}-\lambda_{k}\right|\left|f\left(t^{k}, t^{k+1}\right)\right|}
\end{aligned}
$$


Since (3.6), $a_{k+1}-a_{k} \rightarrow 0$ as $k \rightarrow \infty, g$ is contractive on $C$, Lemma 2.3, Step 2 and the definition of $x^{k+1}$ that $x^{k+1}=a_{k} g\left(x^{k}\right)+a_{k} S\left(t^{k}\right)$, we have

$$
\begin{aligned}
\left\|x^{k+1}-x^{k}\right\|= & \left\|\alpha_{k} g\left(x^{k}\right)+\alpha_{k} S\left(t^{k}\right)-\alpha_{k-1} g\left(x^{k-1}\right)-\alpha_{k-1} S\left(t^{k-1}\right)\right\| \\
= & \|\left(\alpha_{k}-\alpha_{k-1}\right)\left(g\left(x^{k-1}\right)-S\left(t^{k-1}\right)\right)+\left(1-\alpha_{k}\right)\left(S\left(t^{k}\right)-S\left(t^{k-1}\right)\right) \\
& +\alpha_{k}\left(g\left(x^{k}\right)-g\left(x^{k-1}\right)\right) \| \\
\leq & \left|\alpha_{k}-\alpha_{k-1}\right|\left\|g\left(x^{k-1}\right)-S\left(t^{k-1}\right)\right\|+\left(1-\alpha_{k}\right)\left\|t^{k}-t^{k-1}\right\|+\alpha_{k} \delta\left\|x^{k}-x^{k-1}\right\| \\
\leq & \left|\alpha_{k}-\alpha_{k-1}\right|\left\|g\left(x^{k-1}\right)-S\left(t^{k-1}\right)\right\|+\left(1-\alpha_{k}\right)\left(\left\|x^{k}-x^{k-1}\right\|\right. \\
& +\sqrt{\left.2\left|\lambda_{k}-\lambda_{k-1}\right|\left|f\left(t^{k-1}, t^{k}\right)\right|\right)}+\alpha_{k} \delta|| x^{k}-x^{k-1} \| \\
= & \left(1-(1-\delta) \alpha_{k}\right)|| x^{k}-x^{k-1}||+\left|\alpha_{k}-\alpha_{k-1}\right|\left\|g\left(x^{k-1}\right)-S\left(t^{k-1}\right)\right\| \\
& +\left(1-\alpha_{k}\right) \sqrt{2\left|\lambda_{k}-\lambda_{k-1}\right|\left|f\left(t^{k-1}, t^{k}\right)\right|} \\
\leq & \left(1-(1-\delta) \alpha_{k}\right) \| x^{k}-x^{k-1}||+M\left|\alpha_{k}-\alpha_{k-1}\right|+K\left(1-\alpha_{k}\right) \sqrt{2\left|\lambda_{k}-\lambda_{k-1}\right|},
\end{aligned}
$$

where $\delta$ is contractive constant of the mapping $g, M=\sup \left\{\left\|g\left(x^{k-1}\right)-S\left(t^{k-1}\right)\right\|: k=\right.$ $0,1, \ldots\}$ and $K=\sup \left\{\sqrt{\left|f\left(t^{k-1}, t^{k}\right)\right|}: k=0,1, \ldots\right\}$, since $\sum_{k=0}^{\infty}\left|\alpha_{k}-\alpha_{k-1}\right|<\infty$ and $\sum_{k=0}^{\infty} \sqrt{\left|\lambda_{k}-\lambda_{k-1}\right|}<\infty$, in view of Lemma 2.1, we have $\lim _{k \rightarrow \infty}|| x^{k+1}-x^{k} \|=0$.

Step 3. Claim that

$$
\lim _{k \rightarrow \infty}\left\|t^{k}-S\left(t^{k}\right)\right\|=0 .
$$

Proof of Step 3. From $x^{k+1}=a_{k} g\left(x^{k}\right)+\left(1-a_{k}\right) S\left(t^{k}\right)$, we have

$$
\begin{aligned}
x^{k+1}-x^{k} & =\alpha_{k} g\left(x^{k}\right)+\left(1-\alpha_{k}\right) S\left(t^{k}\right)-x^{k} \\
& =\alpha_{k}\left(g\left(x^{k}\right)-x^{k}\right)+\left(1-\alpha_{k}\right)\left(t^{k}-x^{k}\right)+\left(1-\alpha_{k}\right)\left(S\left(t^{k}\right)-t^{k}\right)
\end{aligned}
$$

and hence

$$
\left(1-\alpha_{k}\right)\left\|S\left(t^{k}\right)-t^{k}\right\| \leq\left\|x^{k+1}-x^{k}\right\|+\alpha_{k}\left\|g\left(x^{k}\right)-x^{k}\right\|+\left(1-\alpha_{k}\right)\left\|t^{k}-x^{k}\right\| .
$$

Using this, $\lim _{k \rightarrow \infty} \alpha_{k}=0$, Step 1 and Step 2, we have

$$
\lim _{k \rightarrow \infty}\left\|t^{k}-S\left(t^{k}\right)\right\|=0 .
$$

Step 4. Claim that

$$
\limsup _{k \rightarrow \infty}\left\langle x^{*}-g\left(x^{*}\right), S\left(t^{k}\right)-x^{*}\right\rangle \geq 0 .
$$

Proof of Step 4. By Step 1, $\left\{t^{\natural}\right\}$ is bounded, there exists a subsequence $\left\{t^{k_{i}}\right\}$ of $\left\{t^{\natural}\right\}$ such that

$$
\limsup _{k \rightarrow \infty}\left\langle x^{*}-g\left(x^{*}\right), t^{k}-x^{*}\right\rangle=\lim _{i \rightarrow \infty}\left\langle x^{*}-g\left(x^{*}\right), t^{k_{i}}-x^{*}\right\rangle .
$$

Since the sequence $\left\{t^{k_{i}}\right\}$ is bounded, there exists a subsequence $\left\{t^{k_{i j}}\right\}$ of $\left\{t^{k_{i}}\right\}$ which converges weakly to $\bar{t}$. Without loss of generality we suppose that the sequence $\left\{t^{k_{i}}\right\}$ converges weakly to $\bar{t}$ such that 


$$
\limsup _{k \rightarrow \infty}\left\langle x^{*}-g\left(x^{*}\right), t^{k}-x^{*}\right\rangle=\lim _{i \rightarrow \infty}\left\langle x^{*}-g\left(x^{*}\right), t^{k_{i}}-x^{*}\right\rangle .
$$

Since Lemma 2.2 and Step 3, we have

$$
S(\bar{t})=\bar{t} \Leftrightarrow \bar{t} \in \operatorname{Fix}(S) .
$$

Now we show that $\bar{t} \in \operatorname{Sol}(f, C)$. By Step 1 , we also have

$$
x^{k_{i}} \rightarrow \bar{t}, y^{k_{i}} \rightarrow \bar{t}
$$

Since $y^{k}$ is the unique solution of the strongly convex problem

$$
\min \left\{\frac{1}{2}\left\|y-x^{k}\right\|^{2}+f\left(x^{k}, y\right): y \in C\right\}
$$

we have

$$
0 \in \partial_{2}\left(\lambda_{k} f\left(x^{k}, y\right)+\frac{1}{2}\left\|y-x^{k}\right\|^{2}\right)\left(y^{k}\right)+N_{C}\left(y^{k}\right)
$$

This follows that

$$
0=\lambda_{k} w+y^{k}-x^{k}+w^{k},
$$

where $w \in \partial_{2} f\left(x^{k}, y^{k}\right)$ and $w^{k} \in N_{C}\left(y^{k}\right)$. By the definition of the normal cone $N_{C}$, we have

$$
\left\langle y^{k}-x^{k}, y-y^{k}\right\rangle \geq \lambda_{k}\left\langle w, y^{k}-y\right\rangle, \forall y \in C
$$

On the other hand, since $f\left(x^{k}, \cdot\right)$ is subdifferentiable on $C$, by the well-known Moreau-Rockafellar theorem, there exists $w \in \partial_{2} f\left(x^{k}, y^{k}\right)$ such that

$$
f\left(x^{k}, y\right)-f\left(x^{k}, y^{k}\right) \geq\left\langle w, y-y^{k}\right\rangle, \forall y \in C .
$$

Combining this with (3.9), we have

$$
\lambda_{k}\left(f\left(x^{k}, y\right)-f\left(x^{k}, y^{k}\right)\right) \geq\left\langle y^{k}-x^{k}, y^{k}-y\right\rangle, \forall y \in C .
$$

Hence

$$
\lambda_{k_{j}}\left(f\left(x^{k_{j}}, y\right)-f\left(x^{k_{j}}, \gamma^{k_{j}}\right)\right) \geq\left\langle y^{k_{j}}-x^{k_{j}}, y^{k_{j}}-y\right\rangle, \quad \forall y \in C .
$$

Then, using $\left\{\lambda_{k}\right\} \subset[a, b] \subset\left(0, \frac{1}{L}\right)$ and the continuity of $f$, we have

$$
f(\bar{t}, y) \geq 0, \forall y \in C \text {. }
$$

Combining this and (3.8), we obtain

$$
t^{k_{i}} \rightarrow \bar{t} \in \operatorname{Fix}(S) \cap \operatorname{Sol}(f, C) .
$$

By (3.7) and the definition of $x^{*}$, we have

$$
\limsup _{k \rightarrow \infty}\left\langle x^{*}-g\left(x^{*}\right), t^{k}-x^{*}\right\rangle=\left\langle x^{*}-g\left(x^{*}\right), \bar{t}-x^{*}\right\rangle \geq 0 .
$$

Using this and Step 3, we get

$$
\limsup _{k \rightarrow \infty}\left\langle x^{*}-g\left(x^{*}\right), S\left(t^{k}\right)-x^{*}\right\rangle=\left\langle x^{*}-g\left(x^{*}\right), \bar{t}-x^{*}\right\rangle \geq 0 .
$$

Step 5. Claim that the sequences $\left\{x^{k}\right\},\left\{y^{k}\right\}$ and $\left\{t^{k}\right\}$ converge strongly to $x^{*}$. 
Proof of Step 5. Using $x^{k+1}=\alpha_{k} g\left(x^{k}\right)+\left(1-a_{k}\right) S\left(t^{k}\right)$ and Lemma 2.3, we have

$$
\begin{aligned}
\left\|x^{k+1}-x^{*}\right\|^{2}= & \left\|\alpha_{k}\left(g\left(x^{k}\right)-x^{*}\right)+\left(1-\alpha_{k}\right)\left(S\left(t^{k}\right)-x^{*}\right)\right\|^{2} \\
= & \alpha_{k}^{2}\left\|g\left(x^{k}\right)-x^{*}\right\|^{2}+\left(1-\alpha_{k}\right)^{2}\left\|S\left(t^{k}\right)-x^{*}\right\|^{2} \\
& +2 \alpha_{k}\left(1-\alpha_{k}\right)\left\langle g\left(x^{k}\right)-x^{*}, S\left(t^{k}\right)-x^{*}\right\rangle \\
\leq & \alpha_{k}^{2}\left\|g\left(x^{k}\right)-x^{*}\right\|^{2}+\left(1-\alpha_{k}\right)^{2}\left\|x^{k}-x^{*}\right\|^{2} \\
& +2 \alpha_{k}\left(1-\alpha_{k}\right)\left\langle g\left(x^{k}\right)-x^{*}, S\left(t^{k}\right)-x^{*}\right\rangle \\
= & \alpha_{k}^{2}\left\|g\left(x^{k}\right)-x^{*}\right\|^{2}+\left(1-\alpha_{k}\right)^{2}\left\|x^{k}-x^{*}\right\|^{2} \\
& +2 \alpha_{k}\left(1-\alpha_{k}\right)\left\langle g\left(x^{k}\right)-g\left(x^{*}\right), S\left(t^{k}\right)-x^{*}\right\rangle \\
& +2 \alpha_{k}\left(1-\alpha_{k}\right)\left\langle g\left(x^{*}\right)-x^{*}, S\left(t^{k}\right)-x^{*}\right\rangle \\
\leq & \alpha_{k}^{2}\left\|g\left(x^{k}\right)-x^{*}\right\|^{2}+\left(1-\alpha_{k}\right)^{2}\left\|x^{k}-x^{*}\right\|^{2} \\
& +2 \delta \alpha_{k}\left(1-\alpha_{k}\right)\left\|x^{k}-x^{*}\right\|\left\|\left(t^{k}\right)-x^{*}\right\| \\
& +2 \alpha_{k}\left(1-\alpha_{k}\right)\left\langle g\left(x^{*}\right)-x^{*}, S\left(t^{k}\right)-x^{*}\right\rangle \\
\leq & \alpha_{k}^{2}\left\|g\left(x^{k}\right)-x^{*}\right\|^{2}+\left(\left(1-\alpha_{k}\right)^{2}+2 \delta \alpha_{k}\left(1-\alpha_{k}\right)\right)\left\|x^{k}-x^{*}\right\|^{2} \\
& +2 \alpha_{k}\left(1-\alpha_{k}\right)\left\langle g\left(x^{*}\right)-x^{*}, S\left(t^{k}\right)-x^{*}\right\rangle \\
\leq & \left(1-\alpha_{k}+2 \delta \alpha_{k}\right)\left\|x^{k}-x^{*}\right\|^{2}+\alpha_{k}^{2}\left\|g\left(x^{k}\right)-x^{*}\right\|^{2} \\
& +2 \alpha_{k}\left(1-\alpha_{k}\right) \max \left\{0,\left\langle g\left(x^{*}\right)-x^{*}, S\left(t^{k}\right)-x^{*}\right\rangle\right\} \\
= & \left(1-A_{k}\right)\left\|x^{k}-x^{*}\right\|^{2}+B_{k}
\end{aligned}
$$

where $A_{k}$ and $B_{k}$ are defined by

$$
\left\{\begin{array}{l}
A_{k}=\alpha_{k}(1-2 \delta) \\
B_{k}=\alpha_{k}^{2}\left\|g\left(x^{k}\right)-x^{*}\right\|^{2}+2 \alpha_{k}\left(1-\alpha_{k}\right) \max \left\{0,\left\langle g\left(x^{*}\right)-x^{*}, S\left(t^{k}\right)-x^{*}\right\rangle\right\} .
\end{array}\right.
$$

Since $\lim _{k \rightarrow \infty} \alpha_{k}=0, \sum_{k=1}^{\infty} \alpha_{k}=\infty$, Step 4, we have $\limsup _{k \rightarrow \infty}\left\langle x^{*}-g\left(x^{*}\right), S\left(t^{k}\right)-x^{*}\right\rangle \geq 0$ and hence

$$
B_{k}=o\left(A_{k}\right), \lim _{k \rightarrow \infty} A_{k}=0, \sum_{k=1}^{\infty} A_{k}=\infty .
$$

By Lemma 2.1, we obtain that the sequence $\left\{x^{k}\right\}$ converges strongly to $x^{*}$. It follows from Step 1 that the sequences $\left\{y^{k}\right\}$ and $\left\{t^{k}\right\}$ also converge strongly to the same solution $x^{*}=\operatorname{Pr}_{F i x(S) \cap S o l(f, C)} g\left(x^{* *}\right)$.

$\square$

\section{Applications}

Let $C$ be a nonempty closed convex subset of a real Hilbert space $\mathcal{H}$ and $F$ be a function from $C$ into $\mathcal{H}$. In this section, we consider the variational inequality problem which is presented as follows:

$$
\text { Find } x^{*} \in C \text { such that }\left\langle F\left(x^{*}\right), x-x^{*}\right\rangle \geq 0 \text { for all } x \in C . \quad \operatorname{VI}(F, C)
$$

Let $f: C \times C \rightarrow \mathcal{R}$ be defined by $f(x, y)=\langle F(x), y-x\rangle$. Then Problem $E P(f, C)$ can be written in $\operatorname{VI}(F, C)$. The set of solutions of $\operatorname{VI}(F, C)$ is denoted by $\operatorname{Sol}(F, C)$. Recall that the function $F$ is called strongly monotone on $C$ with $\beta>0$ if

$$
\langle F(x)-F(y), x-y\rangle \geq \beta\|x-y\|^{2}, \forall x, y \in C ;
$$


monotone on $C$ if

$$
\langle F(x)-F(y), x-y\rangle \geq 0, \forall x, y \in C ;
$$

pseudomonotone on $C$ if

$$
\langle F(y), x-y\rangle \geq 0 \Rightarrow\langle F(x), x-y\rangle \geq 0, \forall x, y \in C ;
$$

Lipschitz continuous on $C$ with constants $L>0$ if

$$
\|F(x)-F(y)\| \leq L\|x-y\|, \forall x, y \in C .
$$

Since

$$
\begin{aligned}
y^{k} & =\operatorname{argmin}\left\{\lambda_{k} f\left(x^{k}, y\right)+\frac{1}{2}\left\|y-x^{k}\right\|^{2}: y \in C\right\} \\
& =\operatorname{argmin}\left\{\lambda_{k}\left\langle F\left(x^{k}\right), y-x^{k}\right\rangle+\frac{1}{2}\left\|y-x^{k}\right\|^{2}: y \in C\right\} \\
& =\operatorname{Pr}_{C}\left(x^{k}-\lambda_{k} F\left(x^{k}\right)\right),
\end{aligned}
$$

(2.4), (2.5) and Theorem 3.1, we obtain that the following convergence theorem for finding a common element of the set of fixed points of a nonexpansive mapping $S$ and the solution set of problem $V I(F, C)$.

Theorem 4.1 Let $C$ be a nonempty closed convex subset of a real Hilbert space $\mathcal{H}, F$ be a function from $C$ to $\mathcal{H}$ such that $F$ is monotone and L-Lipschitz continuous on $C, g$ : $C \rightarrow C$ is contractive with constant $\delta \in\left(0, \frac{1}{2}\right), S: C \rightarrow C$ be nonexpansive and positive sequences $\left\{a_{k}\right\}$ and $\left\{\lambda_{k}\right\}$ satisfy the following restrictions

$$
\left\{\begin{array}{l}
\sum_{k=0}^{\infty}\left|\alpha_{k+1}-\alpha_{k}\right|<\infty \\
\lim _{k \rightarrow \infty} \alpha_{k}=0 \\
\sum_{k=0}^{\infty} \alpha_{k}=\infty \\
\sum_{k=0}^{\infty} \sqrt{\left|\lambda_{k+1}-\lambda_{k}\right|}<\infty \\
\left\{\lambda_{k}\right\} \subset[a, b] \text { for some } a, b \in\left(0, \frac{1}{L}\right)
\end{array}\right.
$$

Then sequences $\left\{x^{k}\right\},\left\{y^{k}\right\}$ and $\left\{t^{k}\right\}$ generated by

$$
\left\{\begin{array}{l}
y^{k}=\operatorname{Pr}_{C}\left(x^{k}-\lambda_{k} F\left(x^{k}\right)\right), \\
t^{k}=\operatorname{Pr}_{C}\left(x^{k}-\lambda_{k} F\left(y^{k}\right)\right), \\
x^{k+1}=\alpha_{k} g\left(x^{k}\right)+\left(1-\alpha_{k}\right) S\left(t^{k}\right),
\end{array}\right.
$$

converge strongly to the same point $x^{*} \in \operatorname{Pr}_{F i x(S) \cap S o l(F, C)} g\left(x^{*}\right)$.

Thus, this scheme and its convergence become results proposed by Nadezhkina and Takahashi in [23]. As direct consequences of Theorem 3.1, we obtain the following corollary.

Corollary 4.2 Suppose that Assumptions $A_{1}-A_{3}$ are satisfied, Sol $(f, C) \neq \varnothing, x^{0} \in C$ and two positive sequences $\left\{\lambda_{k}\right\},\left\{a_{k}\right\}$ satisfy the following restrictions: 


$$
\left\{\begin{array}{l}
\sum_{k=0}^{\infty}\left|\alpha_{k+1}-\alpha_{k}\right|<\infty, \\
\lim _{k \rightarrow \infty} \alpha_{k}=0, \\
\sum_{k=0}^{\infty} \alpha_{k}=\infty, \\
\sum_{k=0}^{\infty} \sqrt{\left|\lambda_{k+1}-\lambda_{k}\right|}<\infty,
\end{array}\right.
$$

$\left\{\lambda_{k}\right\} \subset[a, b]$ for some $a, b \in\left(0, \frac{1}{L}\right)$, where $L=\max \left\{2 c_{1}, 2 c_{2}\right\}$.

Then, the sequences $\left\{x^{k}\right\},\left\{y^{k}\right\}$ and $\left\{t^{k}\right\}$ generated by

$$
\left\{\begin{array}{l}
y^{k}=\operatorname{argmin}\left\{\lambda_{k} f\left(x^{k}, y\right)+\frac{1}{2}\left\|y-x^{k}\right\|^{2}: y \in C\right\} \\
t^{k}=\operatorname{argmin}\left\{\lambda_{k} f\left(y^{k}, y\right)+\frac{1}{2}\left\|y-x^{k}\right\|^{2}: y \in C\right\} \\
x^{k+1}=\alpha_{k} g\left(x^{k}\right)+\left(1-\alpha_{k}\right) t^{k}
\end{array}\right.
$$

where $g: C \rightarrow C$ is a $\delta$-contraction with $0<\delta<\frac{1}{2}$, converge strongly to the same point $x^{*}=\operatorname{Pr}_{\text {Sol }(f, \mathrm{C})} g\left(x^{*}\right)$.

\section{Acknowledgements}

We are very grateful to the anonymous referees for their really helpful and constructive comments that helped us very much in improving the paper.

The work was supported by National Foundation for Science and Technology Development of Vietnam (NAFOSTED).

\section{Author details}

${ }^{1}$ Department of Scientific Fundamentals, Posts and Telecommunications Institute of Technology, Hanoi, Vietnam

${ }^{2}$ Department of Mathematics, Haiphong university, Vietnam

\section{Authors' contributions}

The main idea of this paper is proposed by P.N. Anh. The revision is made by DDT. PNA and DDT prepared the manuscript initially and performed all the steps of proof in this research. All authors read and approved the final manuscript.

\section{Competing interests}

The authors declare that they have no competing interests.

Received: 5 December 2011 Accepted: 28 May 2012 Published: 28 May 2012

\section{References}

1. Blum, E, Oettli, W: From optimization and variational inequality to equilibrium problems. The Math Stud. 63, 127-149 (1994)

2. Mastroeni, G: On auxiliary principle for equilibrium problems. In: Daniele P, Giannessi F, Maugeri A (eds.) Nonconvex Optimization and its Applications. Kluwer Academic Publishers, Dordrecht, The Netherlands (2003)

3. Anh, PN: A logarithmic quadratic regularization method for solving pseudomonotone equilibrium problems. Acta Math Vietnamica. 34, 183-200 (2009)

4. Anh, PN: An LQP regularization method for equilibrium problems on polyhedral. Vietnam J Math. 36, 209-228 (2008)

5. Anh, PN, Kim, JK: Outer approximation algorithms for pseudomonotone equilibrium problems. Comp Math Appl. 61, 2588-2595 (2011). doi:10.1016/j.camwa.2011.02.052

6. Quoc, TD, Anh, PN, Muu, LD: Dual extragradient algorithms to equilibrium Problems. J Glob Optim. 52, 139-159 (2012). doi:10.1007/s10898-011-9693-2

7. Anh, PN: Strong convergence theorems for nonexpansive mappings and Ky Fan inequalities. J Optim Theory Appl (2012). DOI 10.1007/s10957-012-0005-X

8. Anh, PN, Kim, JK, Nam, JM: Strong convergence of an extragradient method for equilibrium problems and fixed point problems. J Korean Math Soc. 49, 187-200 (2012). doi:10.4134/JKMS.2012.49.1.187

9. Anh, PN, Son, DX: A new iterative scheme for pseudomonotone equilibrium problems and a finite family of pseudocontractions. J Appl Math Inform. 29, 1179-1191 (2011)

10. Ceng, LC, Schaible, S, Yao, JC: Implicit iteration scheme with perturbed mapping for equilibrium problems and fixed point problems of finitely many nonexpansive mappings. J Optim Theory Appl. 139, 403-418 (2008). doi:10.1007/ s10957-008-9361-y

11. Chen, R, Shen, X, Cui, S: Weak and strong convergence theorems for equilibrium problems and countable strict pseudocontractions mappings in Hilbert space. J Ineq Appl (2010). DOl:10.1155/2010/474813

12. Wang, S, Cho, YJ, Qin, X: A new iterative method for solving equilibrium problems and fixed point problems for an infinite family of nonexpansive mappings. Fix Point Theory Appl (2010). DOI: 10.1155/2010/165098

13. Wangkeeree, R: An extragradient approximation method for equilibrium problems and fixed point problems of a countable family of nonexpansive mappings. Fix Point Theory Appl (2008). DOl:10.1155/2008/134148 
14. Yao, Y, Liou, YC, Jao, JC: Convergence theorem for equilibrium problems and fixed point problems of infinite family of nonexpansive mappings. Fix Point Theory Appl (2007). DOl:10.1155/2007/64363

15. Yao, Y, Liou, YC, Wu, YJ: An extragradient method for mixed equilibrium problems and fixed point problems. Fix Point Theory Appl (2009). DOl: 10.1155/2009/632819

16. Ceng, LC, Petrusel, A, Lee, C, Wong, MM: Two extragradient approximation methods for variational inequalities and fixed point problems of strict pseudo-contractions. Taiwanese J Math. 13, 607-632 (2009)

17. Takahashi, S, Toyoda, M: Weakly convergence theorems for nonexpansive mappings and monotone mappings. J Optim Theory Appl. 118, 417-428 (2003). doi:10.1023/A:1025407607560

18. Zeng, LC, Yao, JC: Strong convergence theorem by an extragradient method for fixed point problems and variational inequality problems. Taiwanese J Math. 10, 1293-1303 (2010)

19. Takahashi, S, Takahashi, W: Viscosity approximation methods for equilibrium problems and fixed point problems in Hilbert spaces. J Math Anal Appl. 331, 506-515 (2007). doi:10.1016/j.jmaa.2006.08.036

20. Anh, PN: A hybrid extragradient method extended to fixed point problems and equilibrium problems. Optimization (2012). DOl:10.1080/02331934.2011.607497

21. Xu, HK: Viscosity approximation methods for nonexpansive mappings. J Math Anal Appl. 298, 279-291 (2004). doi:10.1016/j.jmaa.2004.04.059

22. Goebel, K, Kirk, WA: Topics on metric fixed point theory. Cambridge University Press, Cambridge, England (1990)

23. Nadezhkina, N, Takahashi, W: Weak convergence theorem by an extragradient method for nonexpansive mappings and monotone mappings. J Optim Theory Appl. 128, 191-201 (2006). doi:10.1007/s10957-005-7564-z

doi:10.1186/1029-242X-2012-116

Cite this article as: PN and DD: A new iterative scheme with nonexpansive mappings for equilibrium problems. Journal of Inequalities and Applications 2012 2012:116.

\section{Submit your manuscript to a SpringerOpen ${ }^{\odot}$} journal and benefit from:

- Convenient online submission

- Rigorous peer review

- Immediate publication on acceptance

- Open access: articles freely available online

- High visibility within the field

- Retaining the copyright to your article

Submit your next manuscript at $\boldsymbol{\wedge}$ springeropen.com 